\title{
Journal of Applied Mathematics and Mechanics
}

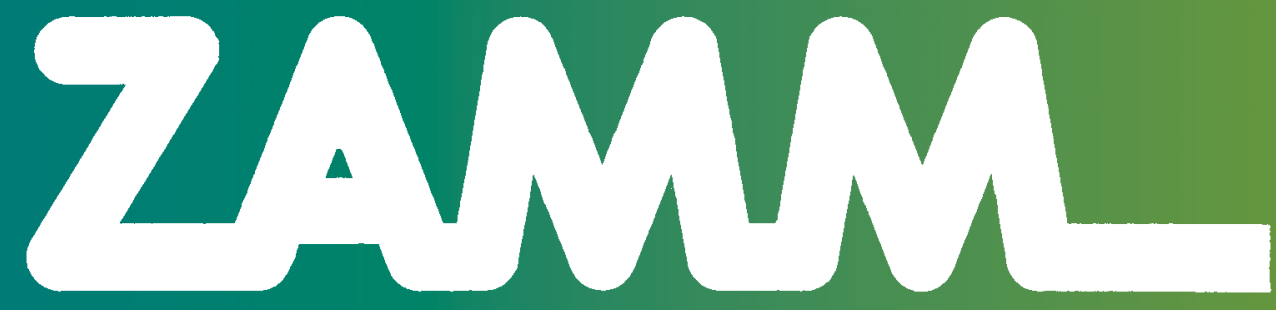

Zeitschrift für Angewandte Mathematik und Mechanik Founded by Richard von Mises in 1921

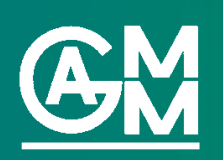

Edited in cooperation with Martin-Luther-Universität Halle-Wittenberg and Gesellschaft für Angewandte

Mathematik und Mechanik e. V. (GAMM)

Editors-in-Chief: H. Altenbach, A. Mielke, S. Odenbach, C. Wieners Managing Editor: H. Altenbach 


\title{
Numerical study of the effect of Navier slip on the driven cavity flow
}

\author{
Qiaolin $\mathrm{He}^{1, *}$ and Xiao-Ping Wang ${ }^{2}$ \\ ${ }^{1}$ Department of Mathematics, The Hong Kong University of Science and Technology, Clear Water Bay, Kowloon, Hong \\ Kong \\ 2 Department of Mathematics and KAUST-HKUST Micro/Nanofludic Joint Laboratory, The Hong Kong University of \\ Science and Technology, Clear Water Bay, Kowloon, Hong Kong
}

Received 3 February 2009, revised 26 April 2009, accepted 20 July 2009

Published online 7 September 2009

Key words Naver-Stokes equation, Navier slip boundary condition, Hopf bifurcation. MSC (2000) 35Q30, 70K50, 76D05, 76D07

\begin{abstract}
We study the driven cavity flow using the Navier slip boundary condition. Our results have shown that the Navier slip boundary condition removes the corner singularity induced by the no-slip boundary condition. In the low Reynolds number case, the behavior of the tangential stress is examined and the results are compared with the analytic results obtained in [14]. For the high Reynolds number, we study the effect of the slip on the critical Reynolds number for Hopf bifurcation. Our results show that the first Hopf bifurcation critical Reynolds number is increasing with slip length.
\end{abstract}

(c) 2009 WILEY-VCH Verlag GmbH \& Co. KGaA, Weinheim

\section{Introduction}

The lid-driven-cavity problem is one of the most important problems in fluid dynamics. It has direct relevance to many of the manufacturing processes in the industry (e.g. coating and mixing). Its importance also results from the fact that it exhibits many of the fundamental features of the flow (shear flow, boundary layers, eddies, core vortex, Hopf bifurcation, and transition to turbulence). Being simple in geometry, it has been widely used as a benchmark problem for numerical methods.

When the no-slip boundary condition is applied at the boundaries, the system exhibits a nonphysical singularity where moving and stationary walls meet. There have been many suggestions for this singularity, for example Koplik and Banavar suggested that the fluid is non-Newtonian in the corner. They first used MD simulations to study the small-scale structure of the driven cavity flow [7]. Their results indicate that slip occurs in the corner region, removing the stress singularity which would otherwise occur. Following this idea, Nie et al. [6] recently develop a multiscale method which couples the MD simulation near the corner and continuum simulation away from the corner to resolve the singularities and determine the singular force.

Another way to remove the singularity is to replace the no-slip boundary condition by a slip boundary condition such as the Navier boundary condition whereby the slip velocity is proportional to the tangential viscous stress and the degree of slip is measured by a slip length [1-4]. The validity of the Navier boundary condition has been shown by Qian and Wang [10] for the driven cavity flow through molecular dynamic and continuum hydrodynamic simulation for a Newtonian fluid. The (constant) slip length is determined from molecular dynamics simulations, and when used as an input for the continuum hydrodynamic simulation it has been shown that molecular dynamics results can be successfully reproduced. It is also shown that the Navier slip will increase the stability threshold for the shear flow [5].

For very small Reynolds number, the Navier Stokes equation is reduced to the linear Stokes equation or the biharmonic equation. The system with the Navier boundary condition can then be solved analytically as a solution of a set of coupled integral equations [14]. In particular, the asymptotic behavior of the tangential stress near the corner can be computed.

For small Reynolds number, the driven cavity flow converges to a stationary state. When the Reynolds number increases over a critical number $\mathrm{Re}_{1}$, the flow becomes periodic in time which indicates a Hopf bifurcation. There exists another critical Reynolds number $\mathrm{Re}_{2}>\mathrm{Re}_{1}$, above which the flow loses time periodicity and becomes chaotic when Reynolds number is large enough. There have been many numerical studies trying to identify the two critical Reynolds numbers $[8,16,17,19,20]$. The most recent simulations by Auteri et al. [9] located the critical Reynolds number $\mathrm{Re}_{1}$ for the first Hopf bifurcation in the interval $[8017.6,8018.8)$.

\footnotetext{
* Corresponding author E-mail: hqlaa@ust.hk
} 
In this paper, we study the effect of the boundary slip on the driven cavity flow numerically using the Navier boundary condition. We carry out numerical simulations using an efficiently numerical method developed in $[12,13,22]$ and we implement the method with the Navier boundary condition. In the low Reynolds number case, the behavior of the tangential stress is examined and the results are compared with the analytic results obtained in [14]. For the high Reynolds number, we study the effect of the slip on the critical Reynolds number for Hopf bifurcation.

\section{Navier slip boundary condition}

The Navier boundary condition is the simplest alternative to the no-slip boundary condition. It states that the amount of slip is proportional to the tangential fluid stress at the solid surface, i.e., $v_{\tau}^{\text {slip }}=-l_{s} \partial_{n} v_{\tau}$, where the proportionality constant $l_{s}$ is the slip length [2-4], which is a phenomenological parameter that measures the local viscous coupling between fluid and solid; $n$ is the normal direction of the solid surface and $v_{\tau}$ is the tangential velocity along the solid surface. Physically a nonzero slip length arises from the unequal wall and fluid densities, the weak wall-fluid interaction, and the high temperature. The verification of the Navier boundary condition in the driven cavity flow has been done in [10] by comparing results from MD simulation and the continuum simulations. The slip length is determined from molecular dynamics simulations and when used as an input for the continuum simulation it has been shown that molecular dynamics results can be successfully reproduced.

\section{Numerical scheme}

The driven cavity flow is governed by the incompressible Navier-Stokes equations

$$
\begin{aligned}
\partial_{t} \vec{u}+(\vec{u} \cdot \nabla) \vec{u}+\nabla p & =\nu \triangle \vec{u}, \\
\nabla \cdot \vec{u} & =0,
\end{aligned}
$$

where $\vec{u}=(u, v)$ are the $x$ and $y$ velocity components, respectively. The computational domain is $\Omega=[0, L] \times[0, L]$ with the top wall moving to the right with the velocity $V_{w}$ (Fig. 1). The boundary condition is

$$
\begin{aligned}
\vec{n} \cdot \vec{u} & =0 \quad \text { on } \quad \Gamma, \\
l_{s}^{-1} \vec{\tau} \cdot \vec{u}^{(s l i p)} & =-\frac{\partial(\vec{\tau} \cdot \vec{u})}{\partial \vec{n}} \quad \text { on } \quad \Gamma,
\end{aligned}
$$

where $\Gamma=\partial \Omega$ and $\vec{n}, \vec{\tau}$ are normal and tangential directions of the boundary $\Gamma$, respectively. We have $\vec{\tau} \cdot \vec{u}($ slip $)=u-V_{w}$ on the top wall (where $V_{w}$ is the speed of the moving wall) and $\vec{\tau} \cdot \vec{u}^{(s l i p)}=\vec{\tau} \cdot \vec{u}$ otherwise.

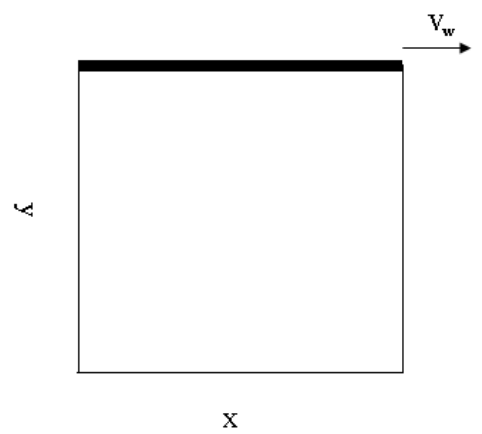

Fig. 1 A cavity flow driven by a moving horizontal wall in the $x y$-plane.

\subsection{Time discretization}

For simulation, we use the method developed in [12,13], and [22]. The time discretization is as follows:

$$
\begin{aligned}
\frac{\vec{u}^{k+1}-\vec{u}^{k}}{\Delta t}-\nu \triangle \vec{u}^{k+1} & =-\vec{u}^{k} \cdot \nabla \vec{u}^{k}-\nabla p^{k}, \\
\Delta p^{k} & =-\nabla \vec{u}^{k}:\left(\nabla \vec{u}^{k}\right)^{T}-\vec{u}^{k} \cdot \nabla\left(\nabla \cdot \vec{u}^{k}\right), \\
\vec{n} \cdot \vec{u}^{k+1} & =0 \text { on } \Gamma,
\end{aligned}
$$




$$
l_{s}^{-1} \vec{\tau} \cdot \vec{u}^{k+1(\operatorname{sip})}=-\frac{\partial\left(\vec{\tau} \cdot \vec{u}^{k+1}\right)}{\partial \vec{n}} \quad \text { on } \Gamma .
$$

The advantage of the scheme is that the convection and pressure terms are treated explicitly and computation of the momentum equation is completely decoupled from the computation of the pressure Poisson equation. Furthermore, the incompressibility is enforced in a more robust way, because the divergence of velocity satisfies a diffusion equation with no-flux boundary conditions. The original method was introduced for no-slip boundary condition and is shown to be unconditionally stable. Here we implement the scheme with the Navier slip boundary condition and our numerical results in Sect. 3.3 show that the method is also unconditionally stable.

\subsection{Space discretization}

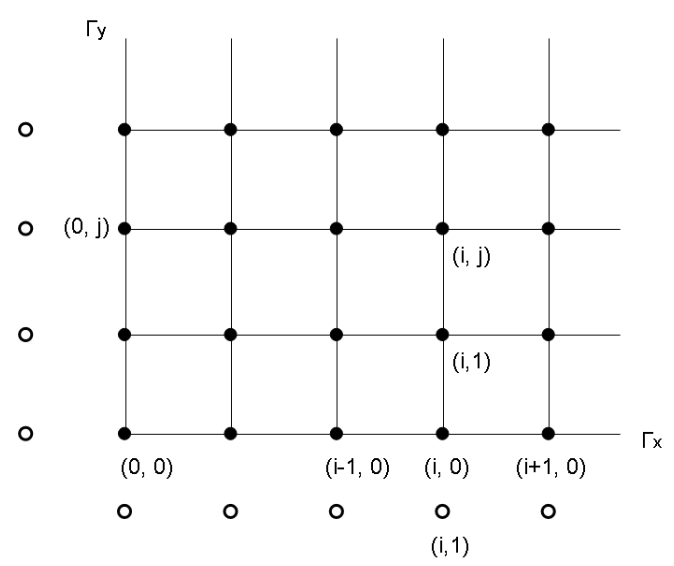

Fig. 2 The computational grid $\Omega_{h}$.

For space discretization, we use a nonstaggered grid shown in Fig. 2. Ghost points (empty circles) are introduced in order to enforce the boundary conditions. We discretize the momentum Eq. (3.5) for $\vec{u}^{k+1}$ with boundary conditions (3.7) and (3.8) by standard second order central difference scheme. For the pressure Poisson equation, we use second-order finite difference scheme on a nonstaggered grid with local pressure boundary condition [13]. We discretize the pressure Poisson equation as following

$$
\triangle_{h} p^{k}=-\nabla_{h} \vec{u}^{k}:\left(\nabla_{h} \vec{u}^{k}\right)^{T}-\vec{u}^{k} \cdot \nabla_{h}\left(\nabla_{h} \cdot \vec{u}^{k}\right)
$$

where $\triangle_{h}, \nabla_{h}$ are the standard second-order centered difference approximation operators. At the grid points $(i, 0)$ along the lower boundary $\Gamma_{x}$ (see Fig. 2), the local pressure boundary condition is derived from (3.5) and (3.7). We then have

$$
\begin{gathered}
\left.\frac{\partial p^{k}}{\partial \vec{n}}\right|_{(i, 0)}=-\left.\frac{\partial p^{k}}{\partial y}\right|_{(i, 0)}=\left.\left(u^{k} v_{x}^{k}+v^{k} v_{y}^{k}\right)\right|_{(i, 0)} \\
-\left.\nu\left(v_{x x}^{k}+v_{y y}^{k}\right)\right|_{(i, 0)}=\left.\nu v_{y y}^{k}\right|_{(i, 0)} .
\end{gathered}
$$

We also have the no-flux boundary condition for the divergence of velocity at the boundary, i.e.

$$
0=\left.\frac{\partial\left(\nabla \cdot \vec{u}^{k}\right)}{\partial \vec{n}}\right|_{(i, 0)}=-\left.\frac{\partial\left(u_{x}^{k}+v_{y}^{k}\right)}{\partial y}\right|_{(i, 0)} \text {. }
$$

Therefore

$$
\left.v_{y y}^{k}\right|_{(i, 0)}=-\left.u_{x y}^{k}\right|_{(i, 0)} .
$$

From the Navier slip boundary condition (3.8), $l_{s}^{-1}\left(u-V_{w u}\right)=u_{y}$ on the lower boundary $(i, 0)$, we have

$$
\left.u_{x y}^{k}\right|_{(i, 0)}=\left.l_{s}^{-1} \frac{\partial\left(u^{k}-V_{w u}\right)}{\partial x}\right|_{(i, 0)} \text {. }
$$

Therefore 


$$
\begin{aligned}
\left.\frac{\partial p^{k}}{\partial \vec{n}}\right|_{(i, 0)} & =-\left.\nu u_{x y}^{k}\right|_{(i, 0)} \\
& =-\nu l_{s}^{-1}\left(\frac{u_{i+1,0}-u_{i-1,0}}{2 h}-\left.\frac{\partial V_{w u}}{\partial x}\right|_{(i, 0)}\right)+O\left(h^{2}\right)
\end{aligned}
$$

for all $0<i<N$. When $i=0$ or $N$ (corner points), we need ghost values $u_{-1,0}, u_{N+1,0}$ which are obtained by enforcing the no-flux boundary condition at the corner points $(0,0)$ and $(N, 0)$. For example, we have

$$
\begin{aligned}
& \left.v_{y y}^{k}\right|_{(i, 0)}=-\left.u_{x y}^{k}\right|_{(i, 0)} \quad \text { on the lower wall, } \\
& \left.u_{x x}^{k}\right|_{(0, j)}=-\left.v_{x y}^{k}\right|_{(0, j)} \quad \text { on the left wall. }
\end{aligned}
$$

From the Navier slip boundary condition, we obtain, at $(0,0)$

$$
\begin{aligned}
& \frac{v_{0,1}-2 v_{0,0}+v_{0,-1}}{h^{2}}=-l_{s}^{-1}\left(\frac{u_{1,0}-u_{-1,0}}{2 h}-\left.\frac{\partial V_{w u}}{\partial x}\right|_{(0,0)}\right), \\
& \frac{u_{1,0}-2 u_{0,0}+u_{-1,0}}{h^{2}}=-l_{s}^{-1}\left(\frac{v_{0,1}-v_{0,-1}}{2 h}-\left.\frac{\partial V_{w v}}{\partial y}\right|_{(0,0)}\right),
\end{aligned}
$$

where $V_{w u}$ is the velocity of boundary $(i, 0)$ and $V_{w v}$ is the velocity of boundary $(0, j)$. Solving $(3.11)$, we can get $u-1,0$ and $v_{0,-1}$.

\subsection{Accuracy check and stability check}

We perform the accuracy check and demonstrate the rate of convergence of the scheme using the following $2 \mathrm{D}$ test case. We also give the stability check.

Example. On a domain $\Omega=[0, \pi] \times[0, \pi]$, we assume a solution of the form

$$
\begin{aligned}
u_{e x}(x, y, t) & =\sin \frac{x}{l_{s}} \cos \frac{y}{l_{s}} F(t), \\
v_{e x}(x, y, t) & =-\cos \frac{x}{l_{s}} \sin \frac{y}{l_{s}} F(t), \\
\frac{\partial p_{e x}}{\partial x} & =-\frac{1}{2 l_{s}} \sin \frac{2 x}{l_{s}} F^{2}(t), \\
\frac{\partial p_{e x}}{\partial y} & =-\frac{1}{2 l_{s}} \sin \frac{2 y}{l_{s}} F^{2}(t), \\
F(t) & =\sin (2 \nu t)
\end{aligned}
$$

which is an exact solution of 2D incompressible N-S equations with a forcing term

$$
\vec{f}=\left(\begin{array}{c}
2 \nu \cos (2 \nu t) \sin \frac{x}{l_{s}} \cos \frac{y}{l_{s}}+\frac{2 \nu}{l_{s}^{2}} \sin \frac{x}{l_{s}} \cos \frac{y}{l_{s}} \sin (2 \nu t) \\
-2 \nu \cos (2 \nu t) \cos \frac{x}{l_{s}} \sin \frac{y}{l_{s}}-\frac{2 \nu}{l_{s}^{2}} \cos \frac{x}{l_{s}} \sin \frac{y}{l_{s}} \sin (2 \nu t)
\end{array}\right)
$$

and satisfy the Navier slip boundary condition:

$$
\begin{aligned}
& v=0, \quad l_{s}^{-1} u^{\text {slip }}=\partial_{y} u \\
& u^{\text {slip }}=u(i, 0)+V_{w}=u(i, 0)-\sin \frac{x(i, 0)}{l_{s}} F(t)
\end{aligned}
$$

along the lower boundary;

$$
v=-\cos \frac{x(i, N)}{l_{s}} \sin \frac{\pi}{l_{s}} F(t), \quad l_{s}^{-1} u^{\text {slip }}=-\partial_{y} u,
$$




$$
u^{\text {slip }}=u(i, N)-V_{w}=u(i, N)-\sin \frac{x(i, N)}{l_{s}} F(t)\left(\cos \frac{\pi}{l_{s}}-\sin \frac{\pi}{l_{s}}\right)
$$

along the upper boundary;

$$
\begin{aligned}
& u=0, \quad l_{s}^{-1} v^{\text {slip }}=\partial_{x} v, \\
& v^{\text {slip }}=v(0, j)+V_{w}=v(0, j)+\sin \left(\frac{y(0, j)}{l_{s}}\right) F(t)
\end{aligned}
$$

along the left boundary;

$$
\begin{aligned}
& u=\cos \frac{y(N, j)}{l_{s}} \sin \frac{\pi}{l_{s}} F(t), \quad l_{s}^{-1} v^{\text {slip }}=-\partial_{x} v, \\
& v^{\operatorname{slip}}=v(N, j)-V_{w}=v(N, j)-\sin \left(\frac{y(N, j)}{l_{s}}\right) F(t)\left(\sin \frac{\pi}{l_{s}}-\cos \frac{\pi}{l_{s}}\right)
\end{aligned}
$$

along the right boundary. Table 1 shows the absolute errors of the numerical solutions compared to the exact solutions. The results clearly indicate the second-order accuracy in $L^{2}$ norm. Fig. 3 shows log-log numerical errors with a fixed $32 \times 32$ mesh and increasing $\Delta t$ from $\Delta t=\frac{1}{32}$ to $\Delta t=8$. The solution and its first derivatives remain of order $O(1)$ up to time $t=1000$. The results indicate first order in time and $\Delta t$ is not restricted by the mesh size in space, which shows the method is unconditionally stable.

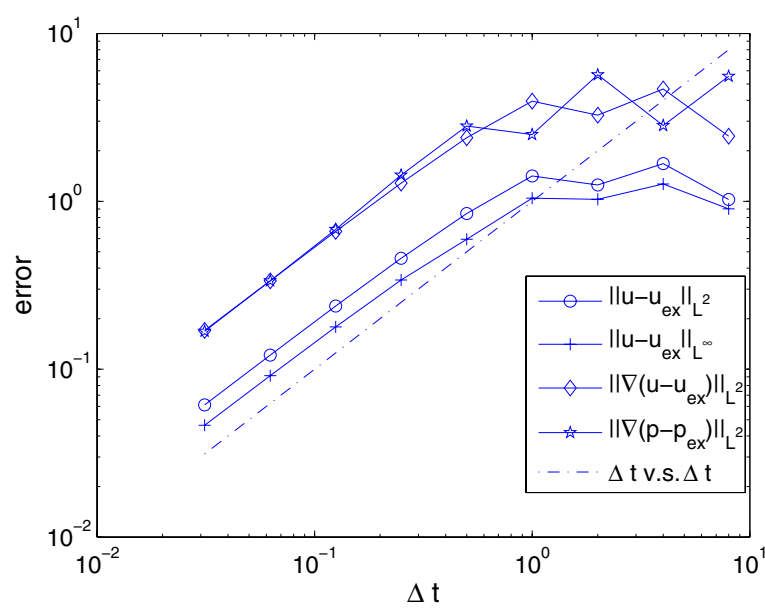

Fig. 3 (online colour at: www.zamm-journal.org) Stability check for scheme (3.5)-(3.8), $\nu=\pi / 5.0, l_{s}=0.5$. Numerical errors in different norms at $t=1000$ for increasing $\Delta t$.

Table 1 Absolute errors at Time $t=1.0, \operatorname{Re}=\pi / \nu=5.0, l_{s}=0.5$.

\begin{tabular}{cccccccc}
\hline & grid points $N$ & $L^{2}$ & order & $H^{1}$ & order & $L^{\infty}$ & order \\
\hline$u$ & $32^{2}$ & $1.5780 \mathrm{E}-02$ & & 0.2417 & & $6.5418 \mathrm{E}-03$ & \\
& $64^{2}$ & $4.0162 \mathrm{E}-03$ & 1.97 & 0.1201 & 1.01 & $1.6722 \mathrm{E}-03$ & 1.97 \\
& $128^{2}$ & $9.9302 \mathrm{E}-04$ & 2.02 & $5.9922 \mathrm{E}-02$ & 1.00 & $3.9407 \mathrm{E}-04$ & 2.08 \\
& $256^{2}$ & $2.4911 \mathrm{E}-04$ & 2.00 & $2.9943 \mathrm{E}-02$ & 1.00 & $9.9478 \mathrm{E}-05$ & 2.00 \\
\hline$v$ & $32^{2}$ & $1.5805 \mathrm{E}-02$ & & 0.2417 & & $6.3651 \mathrm{E}-03$ & \\
& $64^{2}$ & $3.9543 \mathrm{E}-03$ & 2.00 & 0.1201 & 1.01 & $1.5854 \mathrm{E}-03$ & 2.01 \\
& $128^{2}$ & $9.9192 \mathrm{E}-04$ & 2.00 & $5.9921 \mathrm{E}-02$ & 1.00 & $3.9611 \mathrm{E}-04$ & 2.00 \\
& $256^{2}$ & $2.4804 \mathrm{E}-04$ & 2.00 & $2.9943 \mathrm{E}-02$ & 1.00 & $9.9097 \mathrm{E}-05$ & 2.00 \\
\hline$\nabla p$ & $32^{2}$ & 0.3274 & & & & & \\
& $64^{2}$ & 0.1620 & 1.02 & & & & \\
& $128^{2}$ & $8.0644 \mathrm{E}-02$ & 1.01 & & & & \\
& $256^{2}$ & $4.0280 \mathrm{E}-02$ & 1.00 & & & & \\
\hline
\end{tabular}




\section{Numerical results}

It is well known that for driven cavity flow, the Navier-Stokes equations with no-slip boundary condition leads to discontinuity at corners between moving and fixed walls and causes the stress to diverge as the inverse of the distance from the corner $r$. This can be shown asymptotically for small Reynolds numbers when the viscous forces are dominant. The stream function $\psi$ satisfies the biharmonic equation $\nabla^{4} \psi=0$ asymptotically and, for no-slip at the boundaries, a solution may be found which satisfies all the required boundary conditions. Expressions for the pressure and stress or vorticity for the classical solution vary as $r^{-1}$, that is there is a logarithmic singularity when the force on each boundary is calculated. Koplik and Banavar used the MD simulation to study the stress singularity. They observed a break down of the no-slip boundary condition within atomic distances from the corner and a corresponding saturation of the stress. The hybrid multi-scale approach by Nie et al. [6] that treated the singular region atomistically and the reminder of the cavity as a continuum also shows similar results that the stress singularity is cut off near the corner by atomistic effects. In this section, we study the effect of imposing the Navier slip boundary condition for the driven cavity. In particular, we investigate the behavior of the solution near the corner and the long time behavior of the system.

\subsection{The velocity and stress behavior near the corner}

We numerically solve the system (3.1)-(3.4) with different values of $\nu, V_{w}$, and $l_{s}$ but fixed cavity size $L=1$. The system converges to a steady state as $t \rightarrow \infty$ when the Reynolds number $\operatorname{Re}=V_{w} L / \nu$ is not so large, $\operatorname{Re} \leq 1$. In this section, the results are concentrated on the upper-left corner of the cavity. Fig. 4 shows the velocity profiles (scaled with the wall velocity $V_{w}$ and $l_{s}$ ) near the corner for different values of $V_{w}, l_{s}$ and $\nu$. It is evident that there is an universal velocity profile that scales with $V_{w}$ and $l_{s}$ reflecting the fact that when $l_{s}$ is small enough, the system is dominated by the linear Stokes system near the corner. The universal behavior can be explained as follows. Let $\overrightarrow{u_{1}}=\frac{\vec{u}}{V_{w}}, \overrightarrow{x_{1}}=\frac{\vec{x}}{l_{s}}$ and $p_{1}=\frac{l_{s} p}{V_{w}}$, then $\overrightarrow{u_{1}}$ and $p_{1}$ satisfy

$$
\begin{aligned}
l_{s}^{2} \partial_{t} \overrightarrow{u_{1}}+l_{s} V_{w}\left(\overrightarrow{u_{1}} \cdot \nabla\right) \overrightarrow{u_{1}}+\nabla p_{1} & =\nu \Delta \overrightarrow{u_{1}}, \\
\nabla \cdot \overrightarrow{u_{1}} & =0,
\end{aligned}
$$

and the boundary condition $\vec{\tau} \cdot \overrightarrow{u_{1}}(s l i p)=-\frac{\partial\left(\vec{\tau} \cdot \overrightarrow{u_{1}}\right)}{\partial \vec{n}}$. When $l_{s} \rightarrow 0$, the rescaled velocity profile $\overrightarrow{u_{1}}$ satisfies the linear Stokes equation

$$
\nabla p_{1}=\nu \triangle \overrightarrow{u_{1}}
$$

and the slip length $l_{s}$ has been scaled out of the Navier boundary condition.

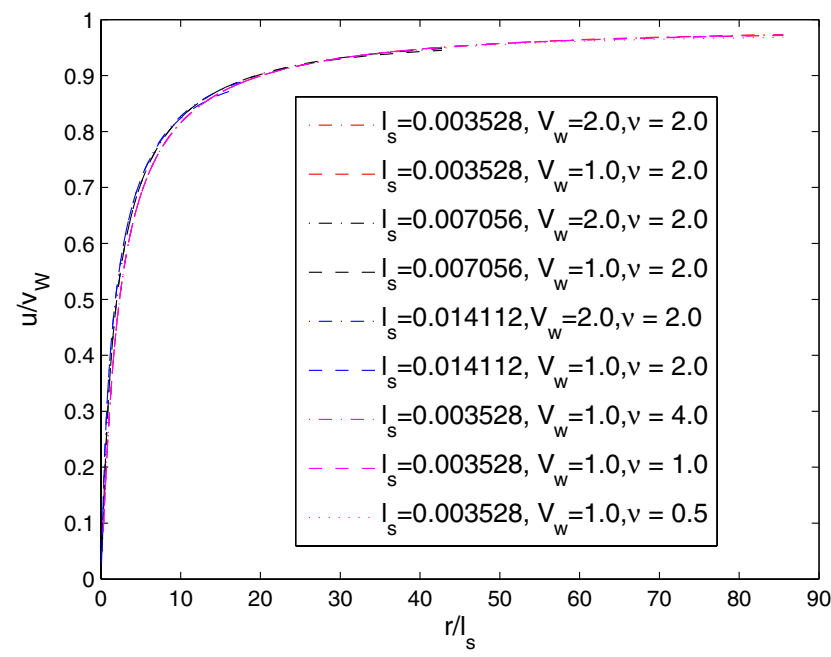

Fig. 4 (online colour at: www.zamm-journal.org) Velocity profiles scaled with the wall velocity $V_{w}, l_{s}$, and $\nu$ near the corner.

The results also show that there is a sharp transition region (of size about $10-20 l_{s}$ ) in which the velocity goes from almost complete slip at the corner to a much slower partial slip region away from the corner and approaches the wall velocity $V_{w}$ as the distance from the corner $r$ becomes large. The behavior of the velocity in the partial slip region is of power law, i.e., $\approx 1 / r$. This power law behavior is similar to that of the slip profile observed in the moving contact line problem [11].

When the Reynolds number is small, the Navier-Stokes equation is reduced to a linear Stokes equation or a biharmonic equation in the stream function formulation. In [14], the authors developed an integral transform solution for solving the 
biharmonic equation $\nabla_{r}^{4} \psi=0$ with the Navier boundary condition, where $\nabla_{r}^{2}=\frac{\partial^{2}}{\partial r^{2}}+\frac{1}{r} \frac{\partial}{\partial r}+\frac{1}{r^{2}} \frac{\partial^{2}}{\partial \theta^{2}}$, which is the polar coordinate form of the biharmonic equation $\nabla^{4} \psi=0$. The Navier slip boundary conditions in polar coordinates have the following form

$$
\begin{aligned}
& \frac{\partial \psi}{\partial r}=0, \quad \frac{1}{r} \frac{\partial \psi}{\partial \theta}=V_{w}-\frac{l_{s}}{r^{2}} \frac{\partial^{2} \psi}{\partial \theta^{2}}, \quad \theta=0, \\
& \frac{\partial \psi}{\partial r}=0, \quad \frac{1}{r} \frac{\partial \psi}{\partial \theta}=\frac{l_{s}}{r^{2}} \frac{\partial^{2} \psi}{\partial \theta^{2}}, \quad \theta=-\frac{\pi}{2} .
\end{aligned}
$$

Their solutions provide the approximation of the tangential stress near the corner

$$
\left.T_{r \theta}\right|_{\theta=0}=\nu V_{w} r^{-1} \frac{\kappa_{a}(\rho)+\kappa_{b}(\rho)}{2}
$$

where $\nu$ is the viscosity, $\rho=\ln \left(r / l_{s}\right)$ and

$$
\begin{aligned}
& \kappa_{a}(\rho)=\frac{2 e^{\rho}}{\pi}\left\{\mathrm{Ci}\left[\left(\frac{\pi+2}{2 \pi}\right) e^{\rho}\right] \sin \left[\left(\frac{\pi+2}{2 \pi}\right) e^{\rho}\right]-\operatorname{si}\left[\left(\frac{\pi+2}{2 \pi}\right) e^{\rho}\right] \cos \left[\left(\frac{\pi+2}{2 \pi}\right) e^{\rho}\right]\right\}, \\
& \kappa_{b}(\rho)=\frac{2 e^{\rho}}{\pi}\left\{\mathrm{Ci}\left[\left(\frac{\pi-2}{2 \pi}\right) e^{\rho}\right] \sin \left[\left(\frac{\pi-2}{2 \pi}\right) e^{\rho}\right]-\operatorname{si}\left[\left(\frac{\pi-2}{2 \pi}\right) e^{\rho}\right] \cos \left[\left(\frac{\pi-2}{2 \pi}\right) e^{\rho}\right]\right\},
\end{aligned}
$$

where $\mathrm{Ci}$ and si are the cosine and sine integrals defined by

$$
\operatorname{Ci}(x)=-\int_{x}^{\infty} \frac{\cos t}{t} d t, \quad \operatorname{si}(x)=-\int_{x}^{\infty} \frac{\sin t}{t} d t .
$$

It is easy to see that as $r$ goes to 0 , the tangential stress on the moving boundary becomes finite, i.e.

$$
\left.T_{r \theta}\right|_{\theta=0} \rightarrow \nu V_{w} / l_{s}
$$

To compare with the asymptotic results, we compute the shear stress for different wall speed $V_{w}$, viscosity $\nu$, and slip length $l_{s}$. Fig. 5 shows the shear stress for different slip lengths (symbols) along the upper boundary and near the upper-left corner. They match well with the asymptotical results. This again confirms that the slip near the corner removes the unphysical singularity of the stress.

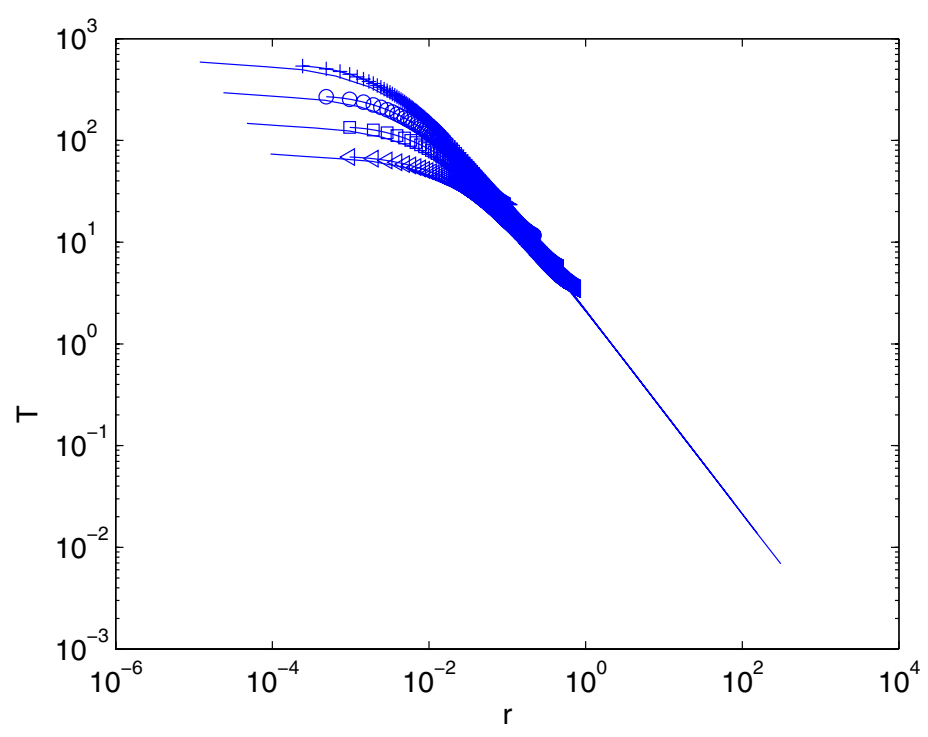

Fig. 5 (online colour at: www.zamm-journal.org) Shear stress $\left.T_{r \theta}\right|_{\theta=0}$ as a function of distance $r$ from the upper-left corner, $\nu=1.0$, computed from $\mathrm{N}-\mathrm{S}$ equations and compared to the asymptotic results. ' + ', ' $\circ$ ', ' $\square$ ', and ' $\triangleleft$ ' represent the results for $l_{s}=0.001764,0.003528,0.007056,0.014112$, respectively. The lines are theoretical results obtained in [14].

The shear stress also demonstrates similar universal behavior after being scaled with the $V_{w}, \nu$, and $l_{s}$. Fig. 6 shows a plot of the rescaled shear stress along the moving wall from the upper-left corner. At the intermediate scales, the stress follows the power law behavior predicted by the Stokes equation. When $r / l_{s}$ is small enough, all results fall onto a common curve that approaches to a constant. The cut-off point $r_{s}$ (deviation from the power law behavior) is at about $10 l_{s}$. 


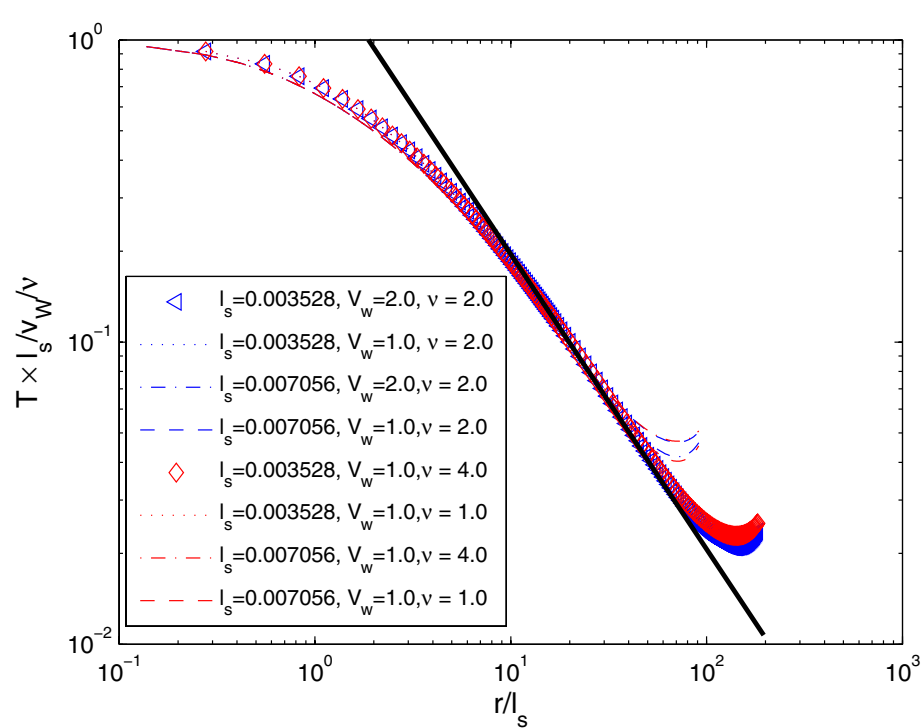

Fig. 6 (online colour at: www.zamm-journal.org) Rescaled shear stress with different $V_{w}, l_{s}$, and $\nu$.

\subsection{Hopf bifurcation}

As the Reynolds number is increased, the driven cavity flow undergoes a sequence of Hopf bifurcations to periodic, quasiperiodic, inverse period-doubling, period-doubling before it reaches a chaotic regime. There is a lot of work on numerical simulations of the Hopf bifurcation phenomena to find the critical Reynolds numbers for bifurcation. For regularized driven cavity flow where the singularity at the upper corners is removed, namely, the horizontal speed on the upper lid of the cavity is taken to be $16 x^{2}(1-x)^{2}$ instead of 1 , Goodrich, Gustafson, and Halasi [15] gave a very detailed investigation at $\operatorname{Re}=5000$. Jie Shen [8] used a Chebyshev-Tau approximation to show that a Hopf bifurcation occurs at a critical Reynolds number in $(10000,10500)$ for the regularized driven cavity flow. Fortin et al. [16] studied the Hopf bifurcations through a classical eigenvalue analysis of the linearized Navier-Stokes equations by means of a finite element spatial discretization. There are many other different results in the literature and the critical Reynolds number $\operatorname{Re}_{c}$ is estimated from 7500 to up to over 10000 [17-21]. Auteri et al. [9] applied a singularity subtraction technique and second-order spectral projection method to locate the critical Reynolds number $\operatorname{Re}_{c}$ for the first Hopf bifurcation in the interval $[8017.6,8018.8$ ), which means the asymptotic solution is steady when Reynolds number is less than $\operatorname{Re}_{c}$ and the asymptotic solution becomes periodic in time when Reynolds number is larger than $\operatorname{Re}_{c}$.

All the numerical studies on Hopf bifurcation so far use no-slip boundary conditions at both moving wall and fixed walls. If the no-slip boundary conditions are replaced by Navier boundary conditions, we have shown in the previous section that the behavior of the solution changes, especially near the corner where the stress singularity is removed. We now want to study how the Navier slip affects the Hopf bifurcation in the lid-driven cavity.

Consider 2D lid-driven cavity problem in $[0,1] \times[0,1]$ with Navier boundary condition and $V_{w}=1.0$ on the top boundary and $V_{w}=0.0$ on the other boundaries. Since the solution has larger gradient near the corner, we use a more refined mesh near the wall to capture the main features of the flow (see Fig. 7). Our purpose is to study how the first

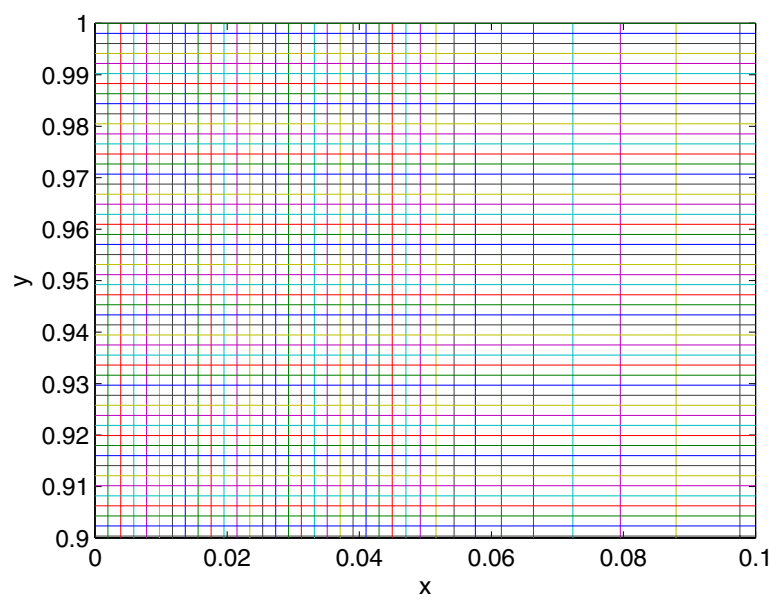

Fig. 7 (online colour at: www.zamm-journal.org) Zoom plot of top left. 
bifurcation Reynolds number changes with slip length. However, it is not our intention to locate exact critical Reynolds number for each slip length since the computational cost is too expensive. Instead, we will locate the critical Reynolds number for each slip length in certain interval.

For several values of slip length, we compute solutions of the $2 \mathrm{D}$ driven cavity flow for increasing Reynolds numbers. We start with $l_{s}=0.003$, our results show that for $\mathrm{Re}=8000$, the solution reaches a steady state after a long transition time. For $\mathrm{Re}=9000$, a stable periodic solution is obtained. This shows that the critical Reynolds number for $l_{s}=0.003$ is between 8000 and 9000. In a similar way, we show that for $l_{s}=0.005, \operatorname{Re}_{c}$ is between 10000 and $10500 ;$ for $l_{s}=0.008$, $\operatorname{Re}_{c}$ is between 11000 and 12000; for $l_{s}=0.01764, \operatorname{Re}_{c}$ is between 16000 and 18000 . It is clear that the critical Reynolds number increases with $l_{s}$.

We now fix a slip length at $l_{s}=0.01764$ and give a detailed description of Hopf bifurcation. For Re $=13000$ and $\mathrm{Re}=16000$, the solution reaches a steady state after a long transition period. This is illustrated in Fig. 8, where the horizontal velocity $u(t)$ at the point $(0.5,0.5)$ is plotted as a function of time.

Fig. 9(a) shows the stream-function for steady state solution at $R e=10000$. It exhibits a large primary vortex with three secondary vortices at three corners. The maximum value of the stream-function of the primary vortex is 0.0688 , which corresponds to $x=0.5273$ and $y=0.5078$. The behavior of the solution near the corners is noticeably different from that of the solution obtained with the no-slip boundary condition (Fig. 9(b)).

At $\operatorname{Re}=18000$, we observe a stable periodic solution for $t$ large enough as shown in Figs. 10 and 11 . In these figures, we plot the time evolution of the first component of the velocity and its power spectrum at the two points $(0.5,0.5)$ and $(0.25,0.81)$. The frequency of the periodic motion is estimated to be roughly $f \approx 0.08$. The behavior is similar for pressure
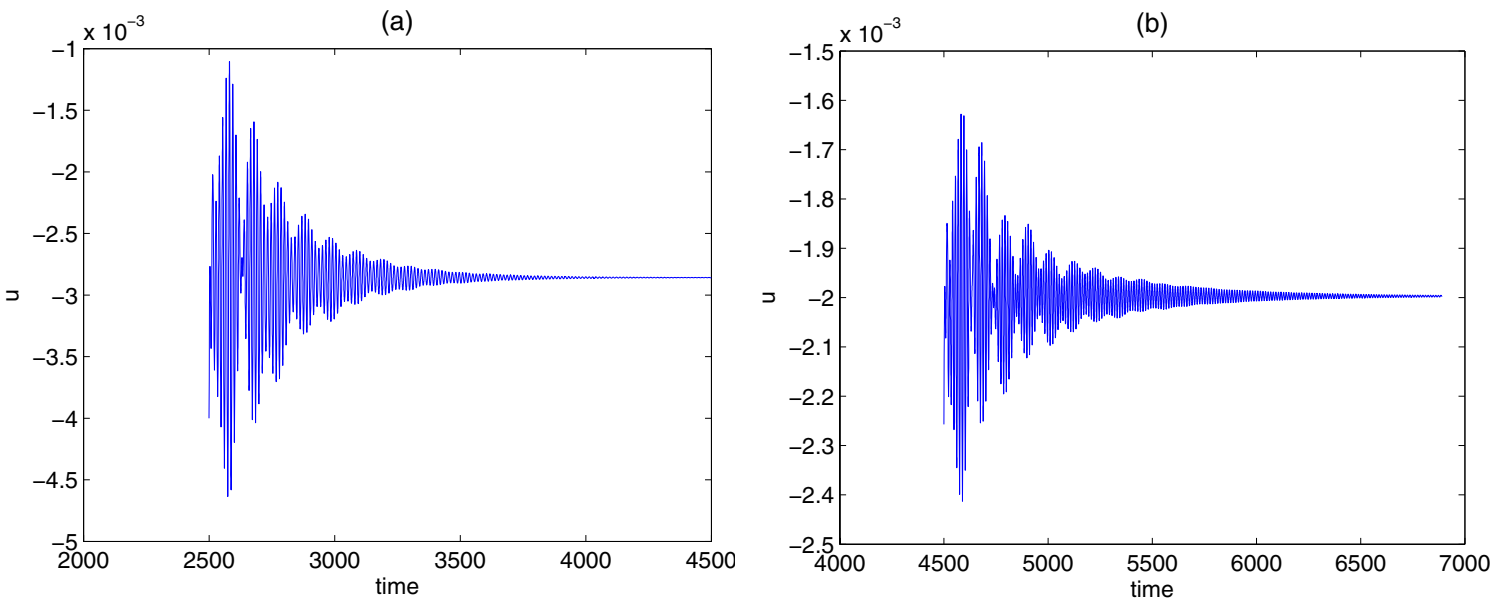

Fig. 8 (online colour at: www.zamm-journal.org) Time evolution of $u$ at point $(0.5,0.5), l_{s}=0.01764$ : (a) $\operatorname{Re}=13000$; (b) $\operatorname{Re}=16000$.

(a)

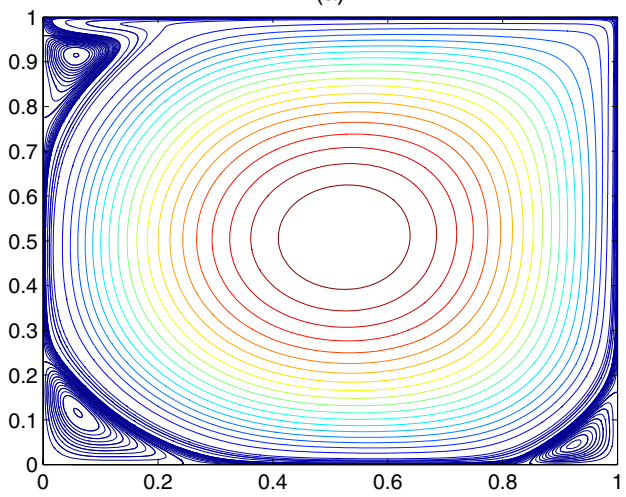

(b)

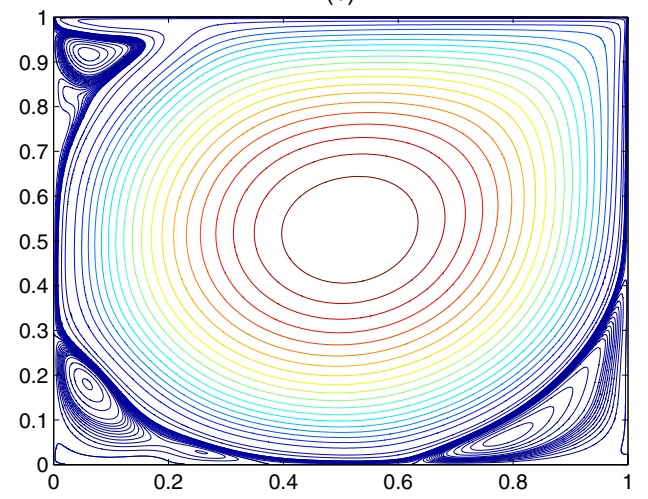

Fig. 9 (online colour at: www.zamm-journal.org) Stream-function at Re $=10000$ with $(a)$ Navier slip boundary condition, $l_{s}=0.01764 ;(b)$ no-slip boundary condition. 

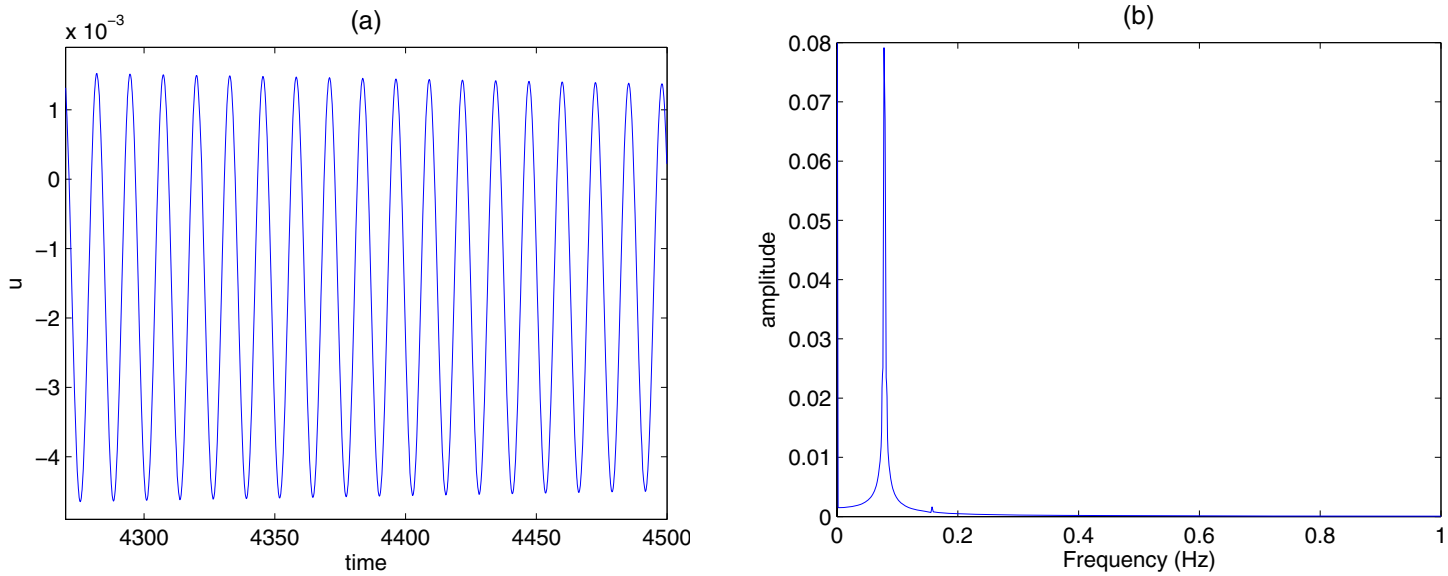

Fig. 10 When $l_{s}=0.01764$, time evolution of $u$ (a) and power spectrum (b) at point $(0.5,0.5)$ for $\operatorname{Re}=18000$.

(a)

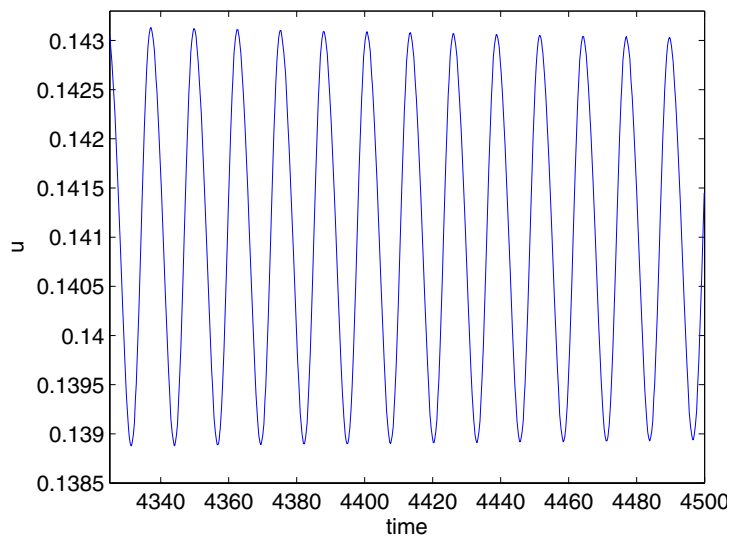

(b)

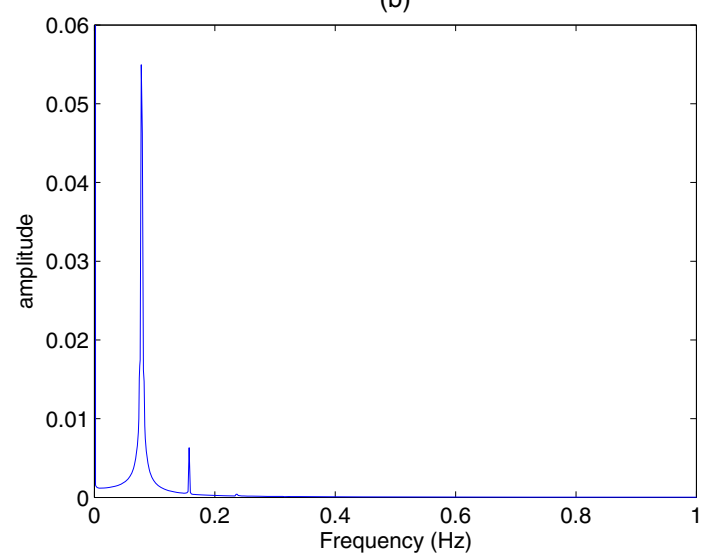

Fig. 11 When $l_{s}=0.01764$, time evolution of $u$ (a) and power spectrum (b) at point $(0.25,0.81)$ for $\operatorname{Re}=18000$.
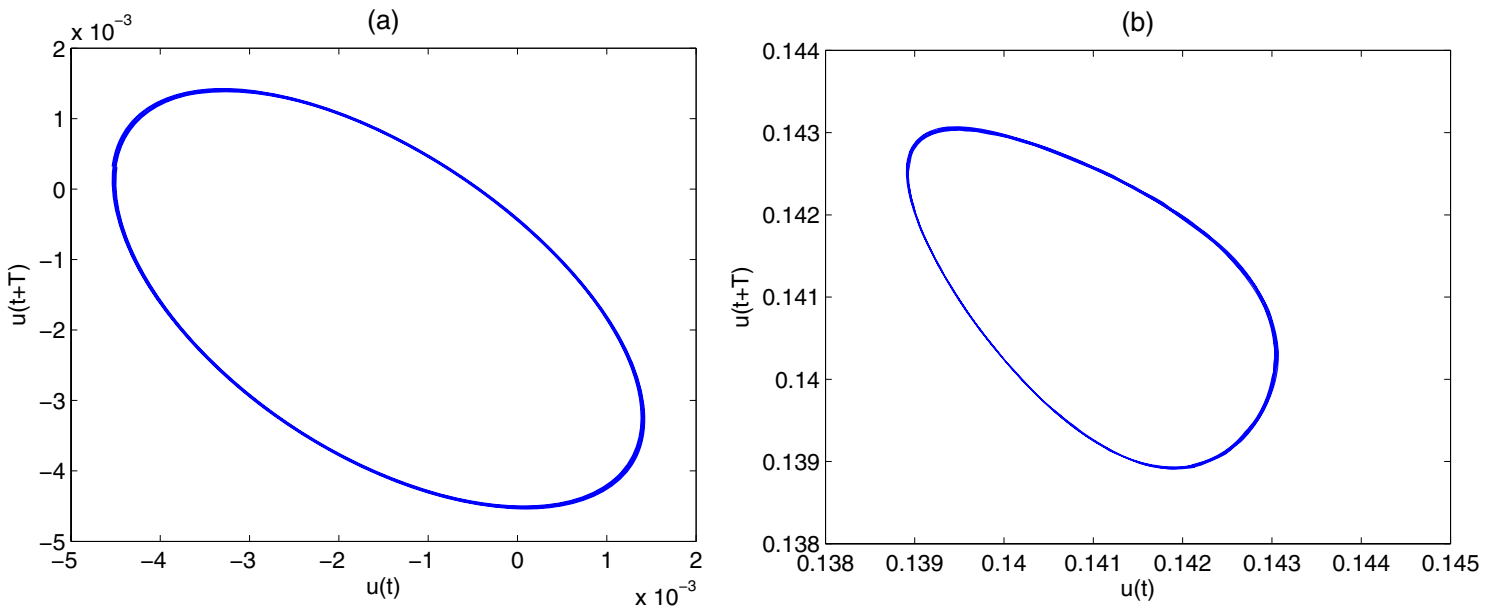

Fig. 12 (online colour at: www.zamm-journal.org) Phase portrait of $u$, where we plot $(u(t), u(t+T))$ for $4300 \leq t \leq$ 4500 and $T=4.4, l_{s}=0.01764$ : (a) at point $(0.5,0.5)$; (b) at point $(0.25,0.81)$. 
(a)

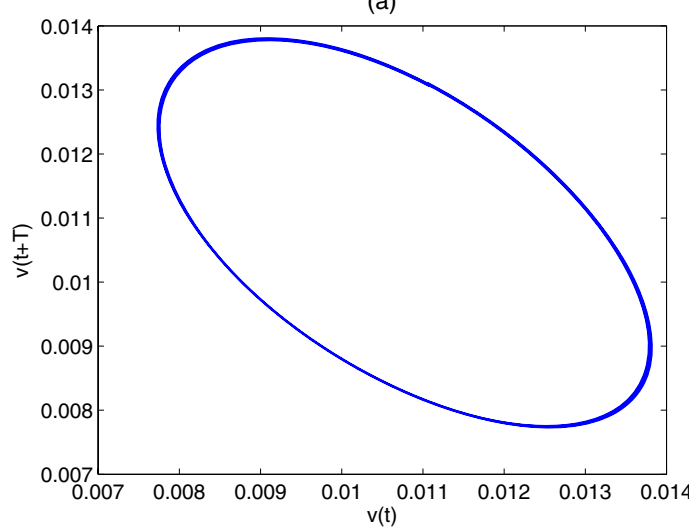

(b)

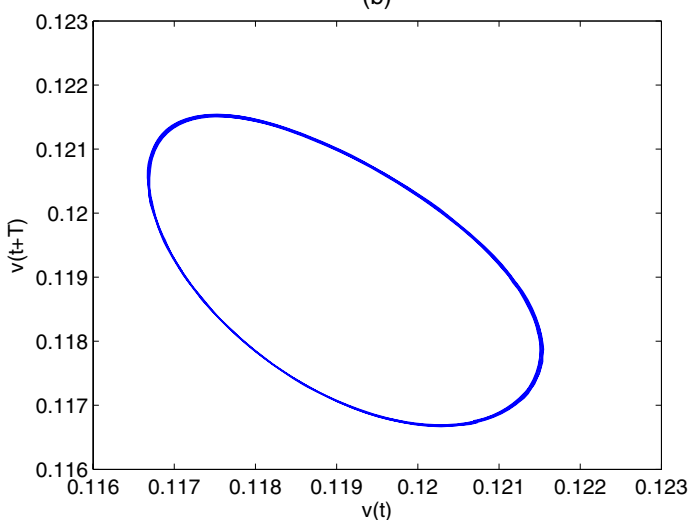

Fig. 13 (online colour at: www.zamm-journal.org) Phase portrait of $v$, where we plot $(v(t), v(t+T))$ for $4300 \leq t \leq$ 4500 and $T=4.4, l_{s}=0.01764$ : (a) at point $(0.5,0.5)$; (b) at point $(0.25,0.81)$.

(a)

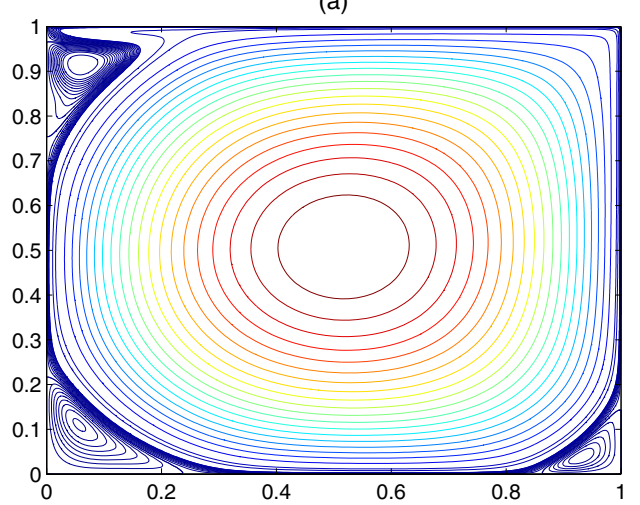

(c)

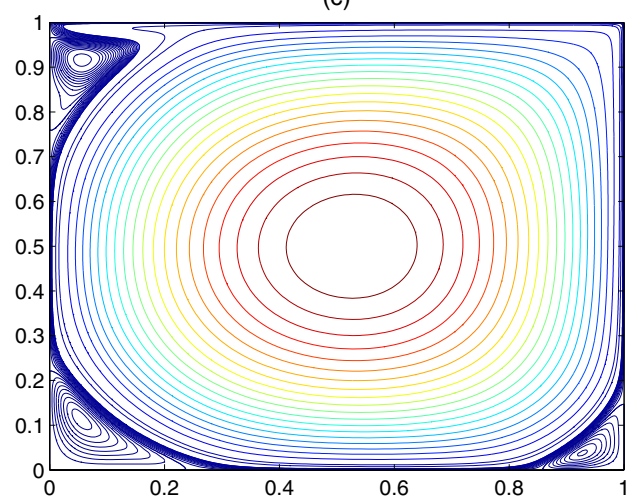

(e)

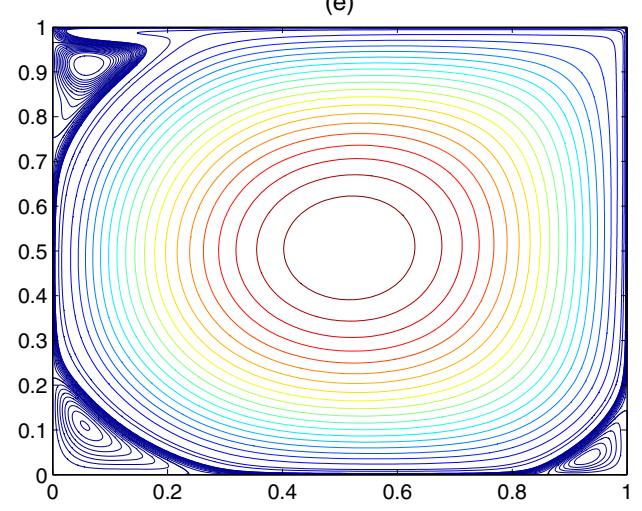

(b)

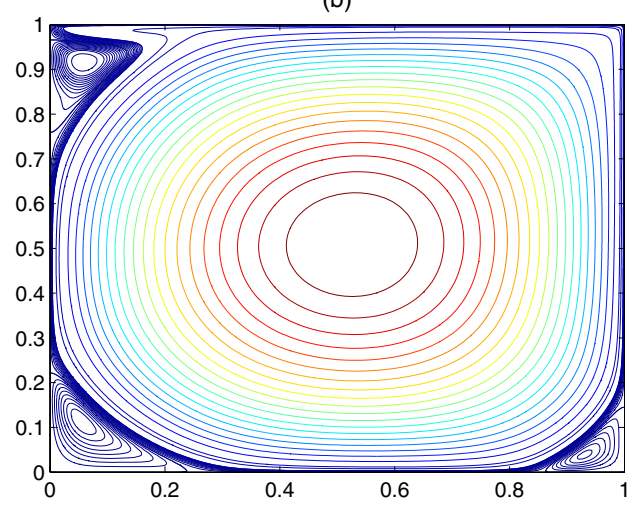

(d)

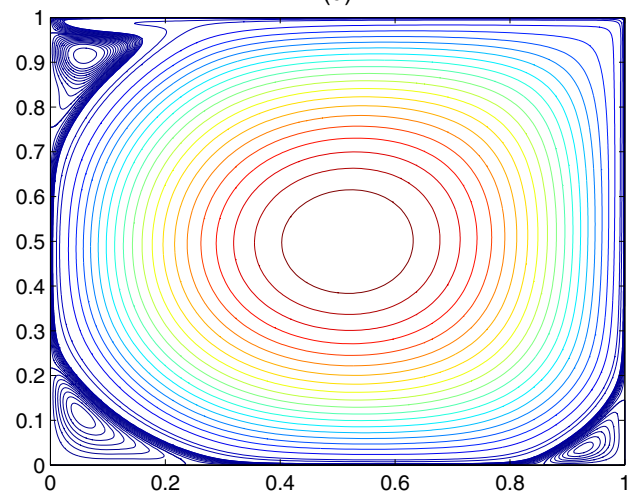

Fig. 14 (online colour at: www.zamm-journal.org) Evolution of the stream function during one main period, $l_{s}=0.01764$ : (a) $t=$ 4502.608; (b) $t=4505.808$; (c) $t=4509.008$; (d) $t=4512.208$; (e) $t=4515.008$. 
and vorticity. The periodicity of the solution is also confirmed by the closed curve of the phase portrait in Figs. 12 and 13. The evolution of the stream function in a complete cycle is given in Fig. 14.

The results have indicated that with the Navier boundary condition and $l_{s}=0.01764$, the first bifurcation occurs at Reynolds number between 16000 and 18000 which is much larger than that with the no-slip boundary conditions. It is clear to see that when $l_{s}$ increases from 0.003 to 0.01764 , the Hopf bifurcation Reynolds number is also increased.

\section{Conclusions}

We have simulated the driven cavity flow using the Navier boundary conditions. We employ a numerical scheme which is implicit in viscosity and explicit in both pressure and convection and use finite difference space discretization with local pressure boundary condition. Our results have shown that the Navier slip boundary condition removes the corner singularity induced by the no-slip boundary condition. The behavior of the shear stress near the corner also agrees with the asymptotic results derived from the biharmonic equations. The Navier slip is also shown to have a strong effect on the Hopf bifurcation of the driven cavity flow. The first Hopf bifurcation critical Reynolds number is significantly increased when slip is allowed.

Acknowledgements This publication is based on work partially supported by Award No. SA-C0040/UK-C0016, made by King Abdullah University of Science and Technology (KAUST), Hong Kong RGC Central Allocation Grant CA05/06.SC01 and RGC-CERG 603107.

\section{References}

[1] P. A. Thompson and M. O. Robbins, Shear flow near solids: Epitaxial and flow boundary conditions, Phys. Rev. A 41, 6830-6837 (1990).

[2] P. A. Thompson and S. M. Troian, A general boundary condition for liquid flow at solid surface, Nature 389, 360-362 (1997).

[3] J.-L. Barrat and L. Bocquet, Large split effect at a nonwetting fluid-solid interface, Phys. Rev. Lett. 82, 4671-4674 (1999).

[4] M. Cieplak and J. R. Banavar, Boundary conditions at a fluid-solid interface, Phys. Rev. Lett. 86, 803-806 (2001).

[5] Q. He and X.-P. Wang, The effect of the boundary slip on the stability of shear flow, Z. Angew. Math. Mech. 88(9), 729-734 (2008).

[6] X. Nie, M. O. Robbin, and S. Chen, Resolving singular forces in cavity flow: Multiscale modeling from atomic to millimeter scales, Phys. Rev. Lett. 96, 134501 (2006).

[7] J. Koplik and J. R. Banavar, Corner flow in the sliding plate problem, Phys. Fluids 7, 3118-3125 (1995).

[8] J. Shen, Hopf bifurcation of the unsteady regularized driven cavity, J. Comput. Phys. 95, 228-245 (1991).

[9] F. Auteri, N. Parolini, and L. Quartapelle, Numerical investigation on the stability of singular driven cavity flow, J. Comput. Phys. 183, 1-25 (2002).

[10] T.Z. Qian and X.P. Wang, Driven cavity flow: from molecular dynamics to continuum hydrodynamics, SIAM Multiscale Model. Simul. 3, 749-763 (2005).

[11] T.Z. Qian, X. P. Wang, and P. Sheng, Power-Law slip profile of the moving contact line in two phase immiscible flows, Phys. Rev. Lett. 63, 094501 (2004).

[12] J. G. Liu, J. Liu, and R. L. Pego, On Incompressible Navier-Stokes Dynamics: a New Approach for Analysis and Computation, Proceedings of the Tenth International Conference on Hyperbolic Problems, HYP2004, held in Osaka, September 2004, (Yokohama Publishers Inc., Osaka, 2004).

[13] H. Johnston and J. G. Liu, Finite difference scheme for imcompressible flow based on local pressure boundary conditions, J. Comput. Phys. 180, 120-154 (2002).

[14] M. T. Matthews and J. M. Hill, Micro/nano sliding plate problem with Navier boundary condition, Z. Angew. Math. Phys. 57, 875-903 (2006).

[15] J. W. Goodrich, K. Gustafson, and K. Halasi, Hopf bifurcation in the driven cavity, J. Comput. Phys. 90, 219-261 (1990).

[16] A. Fortin, M. Jardak, J. Gervais, and R. Pierre, Localization of Hopf bifurcation in fluid flow problems, Int. J. Numer. Methods Fluids 24, 1185-1210 (1997).

[17] C.-H. Bruneau and C. Jouron, An efficient scheme for solving steady incompressible Navier-Stokes equations, J. Comput. Phys. 89, 389-413 (1990).

[18] F. Dexun, M. Yanwen, and L. Hong, Upwind Compact Schemes and Applications, in: Proceedings, 5th Symp. on Comput. Fluid Dyn., vol. 1, Japan Soc. of Comput. Fluid Dyn., 1993.

[19] Y.-F. Peng, Y.-H. Shiau, and R. R. Hwang, Transition in a 2-D lid-driven cavity flow, Comput. Fluids 32, 337-352 (2003).

[20] M. Sahin and R. G. Owens, A novel fully-implicit finite volume method applied to the lid-driven cavity problem. Parts I and II, Int. J. Numer. Methods Fluids 42, 57-77 (2003).

[21] A. Huser and S. Biringen, Calculation of two-dimensional shear-driven cavity flows at high Reynolds number, Int. J. Numer Methods Fluids 14, 1087-1109 (1992).

[22] J.-G. Liu, J. Liu, and R. Pego, Stability and convergence of efficient Navier-Stokes slovers vis a commutator estimate, Commun. Pure Appl. Math. 60, 1443-1487 (2007). 